\title{
On MHD waves, fire-hose and mirror instabilities in anisotropic plasmas
}

\author{
L.-N. Hau and B.-J. Wang \\ Institute of Space Science, National Central University, Jhongli, Taiwan, R.O.C. \\ Received: 11 May 2007 - Revised: 13 August 2007 - Accepted: 13 August 2007 - Published: 3 September 2007
}

\begin{abstract}
Temperature or pressure anisotropies are characteristic of space plasmas, standard magnetohydrodynamic (MHD) model for describing large-scale plasma phenomena however usually assumes isotropic pressure. In this paper we examine the characteristics of MHD waves, fire-hose and mirror instabilities in anisotropic homogeneous magnetized plasmas. The model equations are a set of gyrotropic MHD equations closed by the generalized Chew-Goldberger-Low (CGL) laws with two polytropic exponents representing various thermodynamic conditions. Both ions and electrons are allowed to have separate plasma beta, pressure anisotropy and energy equations. The properties of linear MHD waves and instability criteria are examined and numerical examples for the nonlinear evolutions of slow waves, fire-hose and mirror instabilities are shown. One significant result is that slow waves may develop not only mirror instability but also a new type of compressible fire-hose instability. Their corresponding nonlinear structures thus may exhibit anticorrelated density and magnetic field perturbations, a property used for identifying slow and mirror mode structures in the space plasma environment. The conditions for nonlinear saturation of both fire-hose and mirror instabilities are examined.
\end{abstract}

\section{Introduction}

The magnetohydrodynamic (MHD) model has profound applications in many space and astrophysical plasma problems. Two major assumptions built in the MHD model are the isotropic pressure and the neglect of heat fluxes in the energy equation. In space plasmas the condition of isotropic pressure however is unlikely to be fulfilled due to the lack of sufficient collisions; in particular, in low $\beta$ plasmas the

Correspondence to: L.-N. Hau

(lnhau@jupiter.ss.ncu.edu.tw) pressure tensor usually exhibits the gyrotropic form of $\overleftrightarrow{p}=$ $p_{\|} \hat{b} \hat{b}+p_{\perp}(\overleftrightarrow{I}-\hat{b} \hat{b})$, where $p_{\|}$and $p_{\perp}$ are the pressure component parallel and perpendicular to the magnetic field, respectively, and $\hat{b}=\boldsymbol{B} / \boldsymbol{B}$. In 1956, Chew-Goldberger-Low (Chew et al., 1956) proposed the so-called double-adiabatic or CGL laws, $p_{\perp} / \rho B=C_{\perp}$ and $p_{\|} B^{2} / \rho^{3}=C_{\|}$, as the energy closure for ideal hydromagnetic equations. In the CGLMHD formulation, the plasma is assumed to be a perfect conductor and the heat flux is neglected. The CGL laws were later applied to the subsolar magnetosheath plasma by various authors to test for their validity (e.g., Crooker and Siscoe, 1977; Hau et al., 1993; Hill et al., 1995). The results show that both relations are severely violated and the predicted pressure anisotropy $p_{\perp} / p_{\|}$is far too larger than the observed values. Alternatively, a set of doublepolytropic laws with two empirical polytropic exponents, $p_{\perp} / \rho B^{\gamma_{\perp}-1}=C_{\perp}$ and $p_{\|} B^{\gamma_{\|}-1} / \rho^{\gamma_{\|}}=C_{\|}$, proposed by Hau and Sonnerup (1993), are found to describe the thermodynamics of magnetosheath plasma rather accurately (Hau et al., 1993). The double-polytropic laws may recover the CGL laws for $\gamma_{\|}=3$ and $\gamma_{\perp}=2$ or the double-isothermal condition for $\gamma_{\|}=1$ and $\gamma_{\perp}=1$, and the combination of different $\gamma_{\|}$and $\gamma_{\perp}$ values may be used to describe various thermodynamic states. In the magnetosheath the polytropic values for ions are typically in the range of $\gamma_{\|}=1.14 \pm 0.13$ and $\gamma_{\perp}=0.94 \pm 0.1$ (Hau et al., 1993). The breakdown of the CGL laws may be attributed to the nonideal MHD effects, including imperfect conducting effects and heat fluxes etc. (Hau, 1996).

Hydromagnetic waves with frequencies much lower than the ion gyro-frequency and their corresponding nonlinear structures are often observed in the earth's space plasma environment and interplanetary space (Greenstadt et al., 1968; Tsurutani and Rodriguez, 1981; Tsurutani et al., 1982; Anderson et al., 1990). The analyses of the properties of MHD waves obtained from the double-polytropic MHD equations

Published by Copernicus Publications on behalf of the European Geosciences Union and the American Geophysical Union. 
have shown that for certain parameter regimes slow-mode waves may exhibit anomalous behaviors; for example, slow waves may propagate faster than the intermediate wave and may possess positive density-magnetic field correlation as the fast wave (Hau and Sonnerup, 1993). The anomalies are in contrast with the properties found in the standard isotropic MHD model and have profound implications to various space plasma problems; in particular, the density-magnetic field correlation is often used as a simple rule for distinguishing slow or fast waves and their nonlinear structures in the satellite data. Studies have shown that slow waves with negative density-magnetic field correlation are seldom observed as compared to the fast wave in interplanetary space. This result is usually attributed to the fact that slow waves tend to be damped in the kinetic model (Hada and Kennel, 1985) but an alternative possible explanation is that slow waves with positive density-magnetic field correlation might have been mistaken as the fast wave. Recently the formation of anomalous slow shocks is demonstrated by Hau and Hung (2005) through solving the gyrotropic MHD equations as a Riemann problem; in particular, it is shown that slow shocks may possibly move ahead of intermediate shocks. This result may modify the standard view of magnetic reconnection involving various MHD discontinuities and provide explanation for some anomalous slow-mode structures observed in the geospace plasma environment (Walthour et al., 1994; Whang et al., 1998).

In addition to the MHD waves, two types of low-frequency hydromagnetic instabilities - fire-hose and mirror instabilities may arise in homogeneous anisotropic plasmas that are found to prevail in the solar wind and planetary magnetospheres etc. (e.g., Tsurutani et al., 1982; 1992; Kasper and Lazarus, 2002; Hellinger et al., 2006). The fire-hose and mirror instability criteria based on the CGL-MHD theory are, respectively, to be of $\beta_{\|}-\beta_{\perp}>2$ and $\beta_{\|}<\beta_{\perp}^{2} / 6\left(1+\beta_{\perp}\right)$, and they are associated with the incompressible intermediate and compressible slow mode waves, respectively. The Vlasov theory in hydromagnetic limit however predicts significantly different mirror instability criterion of $\beta_{\|}<\beta_{\perp}^{2} /\left(1+\beta_{\perp}\right)$ (e.g., Hasegawa, 1969). Due to this deficiency, for the last forty years studies of both instabilities are mostly based on the kinetic theory and particle simulations (Kivelson and Southwood, 1996; Quest and Shapiro, 1996; Gary et al., 1998; Pokhotelov et al., 2004). One triumph with the doublepolytropic MHD model is that the predicted mirror instability criterion of $\gamma_{\|} \beta_{\| \mid}<\beta_{\perp}^{2} /\left(2+\gamma_{\perp} \beta_{\perp}\right)$ for $\gamma_{\perp}=2, \gamma_{\|}=1 / 2$ is the same as the kinetic result (Hau and Sonnerup, 1993; Wang and Hau, 2003). In addition to the standard fire-hose instability associated with the incompressible intermediate wave, recently a new type of fire-hose instability associated with the compressible slow wave is identified in the double-polytropic MHD model (Wang and Hau, 2003). In particular, for $\gamma_{\perp}=2$, $\gamma_{\|}=1 / 2$, the instability criteria for the new compressible fire hose are consistent with those obtained from the Vlasov theory in hydromagnetic limit (Hasegawa, 1975). In our double- polytropic MHD formulation, it is also possible to have slow fire hose grow faster than the intermediate fire hose, a result originally found in the kinetic model by Hellinger and Matsumoto (2000). Furthermore, the nonlinear MHD simulation results on fire-hose instabilities are found to be similar to those of the particle simulations; for example, the standard fire-hose instability is found to behave quasi-linearly (Quest and Shapiro, 1996; Gary et al., 1998). These results indicate that the MHD physics may play an important role in the dynamics and evolution of fire-hose instabilities. Note that both kinetic and MHD theories (Hellinger and Matsumoto, 2000; Wang and Hau, 2003) have shown that the standard and new fire-hose instabilities have the maximum growth rates occurring at parallel and oblique propagation angles, respectively, and both parallel and oblique fire-hose instabilities may exist in the solar wind (Hellinger et al., 2006).

The purpose of this paper is to give an overview of the characteristics of MHD waves, fire-hose and mirror instabilities in the double-polytropic MHD model. The gyrotropic MHD formulation used in this study is different from the previous one in that both ions and electrons are treated as separate fluids and may possess different pressure anisotropy, plasma $\beta$ values and energy equations. The two-fluid based MHD formulation is more suitable for modeling the space plasma where ions and electrons may have distinct temperature and pressure anisotropy (e.g., Hau et al., 1993; Phan et al., 1996). Indeed, it has been shown that for the earth's magnetosheath the double-polytropic laws not only provide a viable energy closure for protons but also for electrons with different polytropic exponents though the temperature of electrons is usually much lower than that of ions (Hau et al., 1993). In this study separate pairs of polytropic laws are thus used as energy equations for both ion and electron fluids. The properties of hydromagnetic waves, the conditions for anomalous slow waves and the criteria for fire-hose and mirror instabilities are examined based on the linear doublepolytropic MHD model. The nonlinear evolutions of anomalous slow waves and instabilities are studied based on the gyrotropic MHD simulations; especially, the conditions for nonlinear saturation of fire-hose and mirror instabilities are examined.

\section{Basic equations and linear theory}

The two-fluid equations with neglecting the ion and electron inertia in the electron momentum equation and generalized Ohm's law together with the double-polytropic energy equations for both ions and electrons and Maxwell's equations are as follows.

$$
\begin{aligned}
& \frac{\partial \rho_{i}}{\partial t}+\nabla \cdot \rho_{i} \boldsymbol{u}_{i}=0 \\
& \rho_{i} \frac{d \boldsymbol{u}_{i}}{d t}=-\nabla \cdot\left(\stackrel{\leftrightarrow}{p}_{i}+\stackrel{\leftrightarrow}{p}_{e}\right)+\boldsymbol{j} \times \boldsymbol{B}
\end{aligned}
$$


$\frac{d}{d t}\left(\frac{p_{\perp, s}}{\rho_{s} B^{\gamma_{\perp, s}-1}}\right)=0$

$\frac{d}{d t}\left(\frac{p_{\|, s} B^{\gamma_{\|, s}-1}}{\rho_{s}^{\gamma \|, s}}\right)=0$

$\frac{\partial \boldsymbol{B}}{\partial t}=\nabla \times\left(\boldsymbol{u}_{i} \times \boldsymbol{B}\right)$

$\nabla \times \boldsymbol{B}=\mu_{0} \boldsymbol{j}$

for which the subscripts $i$ and $e$ denote ions and electrons, respectively, while the subscript $s$ can be the ion or electron. Note that in Eq. (2) the electric field has been eliminated from the electron momentum equation, $\nabla \cdot \stackrel{\leftrightarrow}{p}_{e}=n_{e} q_{e}\left(\boldsymbol{E}+\boldsymbol{u}_{e} \times \boldsymbol{B}\right)$, along with the charge neutrality condition of $\sum_{s} n_{s} q_{s}=0$ and the use of Eq. (6) for which the displacement current has been neglected. In Eq. (5), the ion and electron inertia has been assumed zero in the Ohm's law of $\boldsymbol{E}+\boldsymbol{u}_{i} \times \boldsymbol{B}=\left(m_{i} / \rho e\right)\left(\boldsymbol{j} \times \boldsymbol{B}-\nabla \cdot \stackrel{\leftrightarrow}{p}_{e}\right)$ obtained from combining the ion and electron momentum equations. The above two-fluid based MHD formulation is different from the twofluid model in that the inertial effects of both ions and electrons are neglected in the generalized Ohm's law and unlike the standard MHD model the flow velocity as well as the thermal pressure are taken in the individual frame of reference of ions and electrons. However, for cold electron limit the above model has virtually the same form as the previous one-fluid gyrotropic MHD model for which the thermal pressure (also the flow velocity) is taken in the center-of-mass frame of both ions and electrons.

2.1 Dispersion relation and density-magnetic field correlation

Linearization of Eq. (1)-(6) for small-amplitude waves propagating in homogenous magnetized plasmas yields the following dispersion relations for three MHD wave modes.

$$
\begin{aligned}
& \left(\frac{\omega^{2}}{k^{2}}\right)_{i}=\left(1-\sum_{s} \alpha_{s}\right) C_{A}^{2} \cos ^{2} \theta \\
& \left(\frac{\omega^{2}}{k^{2}}\right)_{f, s}=\frac{b \pm \sqrt{b^{2}-4 c}}{2} \\
& b=C_{A}^{2}+\sum_{s}\left[C_{s \perp, s}^{2}+\left(\frac{\gamma_{\|, s}-1}{\gamma_{\|, s}} C_{s \|, s}^{2}-\frac{\gamma_{\perp, s}-1}{\gamma_{\perp, s}} C_{s \perp, s}^{2}\right) \cos ^{2} \theta\right] \\
& c=-\cos ^{2} \theta\left[\sum_{s} C_{s \|, s}^{2}\left(\sum_{s} C_{s \|, s}^{2} \cos ^{2} \theta-b\right)+\right. \\
& \left.\quad \sin ^{2} \theta\left(\sum_{s} \frac{C_{s \perp, s}^{2}}{\gamma_{\perp, s}}\right)^{2}\right] \\
& \text { for } \quad \text { which } C_{A}^{2}=B^{2} / \mu_{0} \rho ; \\
& C_{s \perp, s}^{2}=\gamma_{\perp, s} p_{\perp, s} / \rho ; \quad \alpha_{s}=\mu_{o}\left(p_{\|, s}-p_{\perp, s}\right) / B^{2} ; \quad \text { and } \theta \quad \text { is }
\end{aligned}
$$

the wave propagation angle relative to the background magnetic field. Our dispersion relations are slightly different from those obtained by Hau and Sonnerup (1993) from one-fluid based MHD model in that both ion and electron fluids may have different parameter values, such as the polytropic exponents, plasma $\beta$ and pressure anisotropy. Note that the isotropic MHD dispersion relations may be recovered by substituting $\gamma_{\|, s}=\gamma_{\perp, s}=1$ parametrically and $C_{s \|, i}^{2}=C_{s \perp, i}^{2}=C_{s, i}^{2}=\gamma p_{i} / \rho, \quad C_{s \|, e}^{2}=C_{s \perp, e}^{2}=C_{s, e}^{2}=\gamma p_{e} / \rho$ with $\gamma=5 / 3$ in Eq. (7) and (8). In isotropic MHD model slow waves and fast waves have the unique property of $\delta B \delta \rho<0$ and $\delta B \delta \rho>0$, respectively. In the present model the density-magnetic field correlation has the following form.

$$
\begin{aligned}
\frac{\delta B}{B}= & \frac{a \pm \sqrt{a^{2}+4 \sin ^{2} \theta\left(\sum_{s} C_{s \perp, s}^{2} / \gamma_{\perp s}\right)\left(a+\sum_{s} C_{s \perp, s}^{2} / \gamma_{\perp, s}\right)}}{2\left(a+\sum_{s} C_{s \perp, s}^{2} / \gamma_{\perp . s}\right)} \frac{\delta \rho}{\rho} \\
a= & C_{A}^{2}+\sum_{s}\left(\frac{\gamma_{\perp, s}-2}{\gamma_{\perp, s}}\right) C_{s \perp, s}^{2}+\left[\sum_{s}\left(\frac{3-\gamma_{\perp, s}}{\gamma_{\perp, s}}\right) C_{s \perp, s}^{2}\right. \\
& \left.-\left(\frac{\gamma_{\|, s}+1}{\gamma_{\|, s}}\right) C_{s \|, s}^{2}\right] \cos ^{2} \theta
\end{aligned}
$$

\subsection{Anomalous slow waves}

Examining the dispersion relations, it can be shown that the phase speed of slow waves may be greater than that of intermediate waves for the condition of

$$
\sum_{s}\left(\gamma_{\|, s}+1\right) \beta_{\|, s}>2+\sum_{s} \beta_{\perp, s}+\frac{\left(\sum_{s} \beta_{\perp, s}\right)^{2}}{\left(2+\sum_{s} \gamma_{\perp, s} \beta_{\perp, s}\right)}
$$

Figure 1 shows the threshold condition on $\beta_{\|, i}$ versus $\beta_{\perp, i}$ plot for different pairs of $\left(\gamma_{\|, i}, \gamma_{\perp, i}\right)$ and for $\gamma_{\|, e}=1, \gamma_{\perp, e}=1$, $\beta_{\perp, e}=\beta_{\perp, i} / 10, \beta_{\|, e} / \beta_{\perp, e}=1.5$. The electrons are assumed isothermal due to their mobility that is more or less consistent with the observational result in the magnetosheath (Hau et al., 1993). The regions above and below the threshold curves correspond to $(\omega / k)_{s}>(\omega / k)_{i}$ and $(\omega / k)_{i}>(\omega / k)_{s}$, respectively, and the results for $\beta_{\|, e} / \beta_{\perp, e}=0.5$ appear the same. Note that the region of $\beta_{\|}-\beta_{\perp}>2$ is unstable to fire-hose instability and the condition of $(\omega / k)_{s}>(\omega / k)_{i}$ becomes inappropriate. As for the case of cold electrons, fast waves always have $\delta B \delta \rho>0$, while slow waves may have the anomalous behavior of $\delta B \delta \rho>0$ under the following condition.

$$
\begin{aligned}
& 2+\sum_{s}\left(\gamma_{\perp, s}-1\right) \beta_{\perp, s} \leq \sum_{s}\left[\left(\gamma_{\|, s}+1\right) \beta_{\|, s}\right. \\
& \left.+\left(\gamma_{\perp, s}-3\right) \beta_{\perp, s}\right] \cos ^{2} \theta
\end{aligned}
$$

Figure 2 shows the threshold condition on the $\beta_{\perp, i}$ versus $\theta$ plot for the parameter values of $p_{\perp, i} / p_{\|, i}=0.1,0.5,0.7,0.8$; $\left(\gamma_{\|, i}, \gamma_{\perp, i}\right)=(1,1)$ or $(1,2)$; and isothermal electrons with $\beta_{\|, e}=0.1 \beta_{\|, i}, \beta_{\perp, e} / \beta_{\|, e}=0.5$. It is seen that slow waves with $\delta B \delta \rho>0$ may occur for various parameter regimes. 


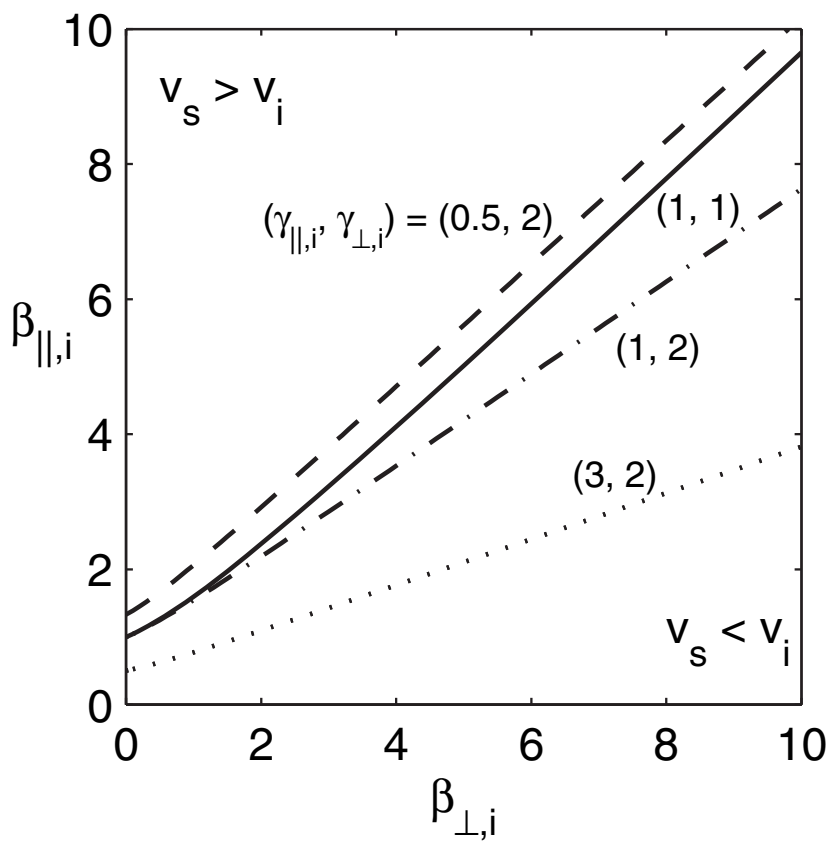

Fig. 1. Critical conditions for slow waves to propagate faster than the intermediate waves for different pairs of $\left(\gamma_{\|, i}, \gamma_{\perp, i}\right)$ and $\gamma_{\|, e}=1, \gamma_{\perp, e}=1, \beta_{\perp, e}=\beta_{\perp, i} / 10, \beta_{\|, e} / \beta_{\perp, e}=1.5$. The regions above and below the threshold curves correspond to $(\omega / k)_{s}>(\omega / k)_{i}$ and $(\omega / k)_{i}>(\omega / k)_{s}$, respectively.

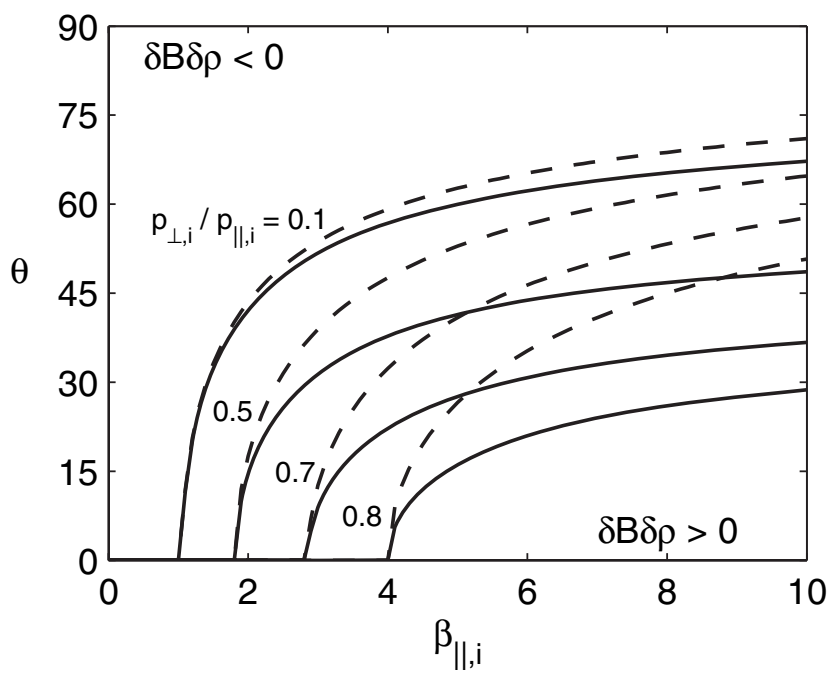

Fig. 2. Critical propagation angle in Eq. (11) versus $\beta_{\|, i}$ for various $p_{\perp, i} / p_{\|, i}$ and $p_{\perp, e} / p_{\|, e}=0.5, \quad \beta_{\|, e}=0.1 \beta_{\|, i}, \quad \gamma_{\perp, e}=1, \gamma_{\|, e}=1$, $\left(\gamma_{\|, i}, \gamma_{\perp, i}\right)=(1,2)$ (solid curves) and $(1,1)$ (dashed curves). The regions above and below each curve correspond to slow waves with $\delta B \delta \rho<0$ and $\delta B \delta \rho>0$, respectively.

\subsection{Fire-hose and mirror instabilities}

It is easily seen from Eq. (7) that the intermediate mode becomes unstable for $\sum_{S}\left(\beta_{\|, s}-\beta_{\perp, s}\right)>2$ which is the standard incompressible fire-hose instability. For slow waves, the unstable conditions ( $c<0$ in Eq. 8) may depend on whether the condition $\beta_{\|}-\beta_{\perp}>2$ is complied or not. For fire-hose stable condition, i.e., $\beta_{\|}-\beta_{\perp}<2$, slow waves are unstable provided that

$$
\sum_{s} \gamma_{\|, s} \beta_{\|, s}<\frac{\left(\sum_{s} \beta_{\perp, s}\right)^{2}}{2+\sum_{s} \gamma_{\perp, s} \beta_{\perp, s}}
$$

and $\theta>\theta_{c}$, where

$$
\theta_{c}=\tan ^{-1}\left[\frac{\sum_{s}\left(\beta_{\|, s}-\beta_{\perp, s}\right)-2}{2+\sum_{s}\left(\gamma_{\perp, s} \beta_{\perp, s}\right)-\left(\sum_{s} \beta_{\perp, s}\right)^{2} / \sum_{s} \gamma_{\|, s} \beta_{\|, s}}\right]^{1 / 2}
$$

Equation (12) is the generalized mirror instability criterion and may also be obtained from taking the limit of $\theta \rightarrow 90^{\circ}$ in the relation of $\left(\omega^{2} / k^{2}\right)_{s}<0$, but it is merely the necessary condition and the criterion $\theta>\theta_{c}$ needs also be complied for slow waves to develop the mirror instability. When $\beta_{\|}-\beta_{\perp}>2$, slow waves can be shown to be unstable for $\theta<\theta_{c}$, i.e., two types of fire-hose instabilities may coexist. Although both mirror and compressible fire-hose instabilities comply with the same threshold propagation angle but the former occurs at more oblique angles while the latter occurs at more parallel angles. As shown in our earlier study for cold electron limit and $\gamma_{\perp, i}=2, \gamma_{\|, i}=1 / 2$, the instability criteria for both mirror and fire-hose instabilities are identical to those obtained from the Vlasov theory. It is clearly that the instability criteria may be modified by the electron temperature anisotropy though the cold electron limit represents a reasonable approximation for certain space plasmas such as the magnetosheath where the electron temperature is much smaller than the ion temperature (e.g., Hau et al., 1993; Phan et al., 1996). Figure 3 shows the growth rate versus $\theta$ for the parameter values of $\beta_{\perp, i}=0.5, \beta_{\|, i}=3, \gamma_{\perp, i}=2, \gamma_{\|, i}=1 / 2$ and $\gamma_{\|, e}=1, \gamma_{\perp, e}=1$ as well as for various electron beta and pressure anisotropy. In all cases the intermediate fire hose grows faster than the slow fire hose.

In our earlier study for cold electron case, it is shown that for $\gamma_{\|, i} \gamma_{\perp, i}<1$ the slow fire hose may grow faster than the intermediate fire hose and has the maximum growth rate occurring at oblique propagation. To achieve this result, several conditions need to be complied: $\beta_{\|}-\beta_{\perp}>2,\left(\omega^{2} / k^{2}\right)_{s}<0$ and $\left(\omega^{2} / k^{2}\right)_{s}<\left(\omega^{2} / k^{2}\right)_{i}$ (i.e., the reverse condition in Eq. 10). In the present model the necessary condition is $\beta_{\perp, i}>\beta_{\perp c}$, where

$$
\begin{aligned}
& \beta_{\perp c}=\frac{-B \pm \sqrt{B^{2}-4 A C}}{2 A} \\
& A=1-\gamma_{\|, i} \gamma_{\perp, i}
\end{aligned}
$$




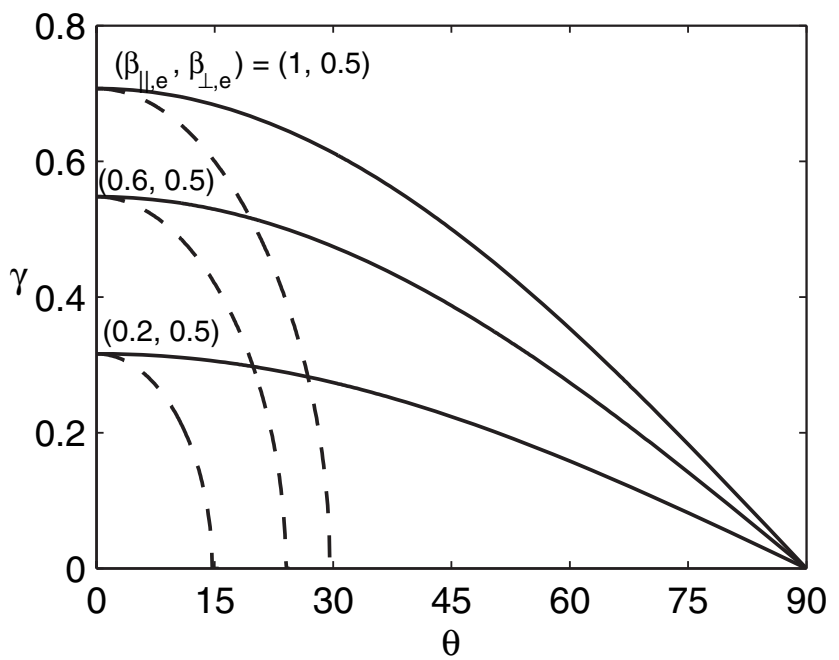

Fig. 3. The growth rate of compressible (dashed curves) and incompressible (solid curves) fire-hose instabilities as function of $\theta$ for the parameter values of $\beta_{\perp, i}=0.5, \beta_{\|, i}=3, \gamma_{\perp, i}=2, \gamma_{\|, i}=1 / 2$, $\gamma_{\|, e}=1, \gamma_{\perp, e}=1$ and for various values of $\left(\beta_{\|, e}, \beta_{\perp, e}\right)$.

$$
\begin{aligned}
B= & -\left[\left(2-\delta \beta_{e}\right) \gamma_{\perp, i} \gamma_{\|, i}+\gamma_{\perp, i} \gamma_{\|, e} \beta_{\|, e}\right. \\
& \left.+\left(\gamma_{\|, i} \gamma_{\perp, i}-2\right) \beta_{\perp, e}+2 \gamma_{\|, i}\right] \\
C= & -\left[\left(2-\delta \beta_{e}\right)\left(\gamma_{\perp, e} \beta_{\perp, e}+2\right) \gamma_{\|, i}\right. \\
& \left.+\left(\gamma_{\perp, e} \beta_{\perp, e}+2\right)\left(\gamma_{\|, e} \beta_{\|, e}\right)-\beta_{\perp, e}^{2}\right]
\end{aligned}
$$

for which $\delta \beta_{e}=\beta_{\|, e}-\beta_{\perp, e}$. For physical plasma beta of $\beta_{\perp c}>0$, there is constraint on the ion polytropic values for given other parameters. Figure 4 shows similar calculations as in Fig. 3 but with different ion polytropic exponents of $\gamma_{\perp, i}=1, \gamma_{\|, i}=1 / 2$ that has $\gamma_{\|, i} \gamma_{\perp, i}<1$. As indicated, the growth rate of compressible fire hose is greater than that of incompressible fire hose with the maximum growth rate being at oblique propagation angles.

In the parameter regime where both conditions of $\beta_{\|}-\beta_{\perp}>2$ and $\gamma_{s}>\gamma_{i}$ are met (where $\gamma_{s}$ and $\gamma_{i}$ are, respectively, to be the growth rate of slow and intermediate firehose instabilities), $\theta_{c}$ in Eq. (13) however is unphysical and therefore the criterion for this particular type of instability is not the same as the regular compressible fire-hose instability. It can be shown that this peculiar type of compressible firehose instability indeed satisfies the generalized mirror condition of $\sum_{s} \gamma_{\|, s} \beta_{\|, s}<\left(\sum_{S} \beta_{\perp, s}\right)^{2} /\left(2+\sum_{s} \gamma_{\perp, s} \beta_{\perp, s}\right)$ and the mode is unstable for all propagation angles with the maximum growth rate being at oblique propagation. In particular, for cold electrons the generalized mirror criterion is $p_{\perp} / p_{\|}>\gamma_{\|}\left(\gamma_{\perp}+2 / \beta_{\perp}\right)$ and the pressure anisotropy $p_{\perp} / p_{\|}$ can possibly be less than one for $\gamma_{\|, i} \gamma_{\perp, i}<1$ and $\beta_{\perp}>2 \gamma_{\|, i}$. Therefore, in the double-polytropic MHD model it is possible to find the parameter regime for which both the firehose $\left(\beta_{\|}-\beta_{\perp}>2\right)$ and generalized mirror instability condi-



Fig. 4. The growth rate of compressible (dashed curves) and incompressible (solid curves) fire-hose instabilities as function of $\theta$ for the parameter values of $\beta_{\perp, i}=6, \beta_{\|, i}=8.1, \gamma_{\perp, i}=1, \gamma_{\|, i}=1 / 2$, $\gamma_{\|, e}=1, \gamma_{\perp, e}=1$ and for various values of $\left(\beta_{\|, e}, \beta_{\perp, e}\right)$.

tions $\left(\sum_{s} \gamma_{\|, s} \beta_{\|, s}<\left(\sum_{s} \beta_{\perp, s}\right)^{2} /\left(2+\sum_{s} \gamma_{\perp, s} \beta_{\perp, s}\right)\right)$ are satisfied, giving rise to both standard incompressible and new compressible fire-hose instabilities.

\section{Nonlinear evolution}

In this section we examine the evolution of anomalous slow waves, fire-hose and mirror instabilities based on the numerical simulations of double-polytropic MHD equations. In the nonlinear model it is more convenient to use the double-polytropic energy laws in conservative forms: $\partial S_{\|, s} / \partial t+\nabla \cdot\left(S_{\|, s} \boldsymbol{u}\right)=0$ and $\partial S_{\perp, s} / \partial t+\nabla \cdot\left(S_{\perp, s} \boldsymbol{u}\right)=0$ where $S_{\|, s}=p_{\|, s}\left(B / \rho_{i}\right)^{\gamma_{\|, s}-1}$ and $S_{\perp, s}=p_{\perp, s} / B^{\gamma_{\perp, s}-1}(\mathrm{Hau}$, 2002). The numerical experiments are conducted to examine the nonlinear evolution of stable anomalous slow waves and unstable intermediate and slow waves including fire-hose and mirror instabilities. In the nonlinear phase short wavelengths tend to occur and finite resistivity and/or viscosity need be introduced in the ideal double-polytropic MHD model to provide the necessary dissipation for nonlinear saturation (Wang and Hau, 2003). For simplicity and to reduce the free parameters, the electrons are assumed cold and the nonlinear model is thus similar to that adopted by Wang and Hau (2003) for studying the MHD fire-hose instabilities.

\subsection{Anomalous slow waves}

As shown above, anomalous slow waves with phase speed greater that of the intermediate wave and/or with in-phase density and magnetic field perturbations may exist for a wide range of parameter regimes. One relevant issue is whether 

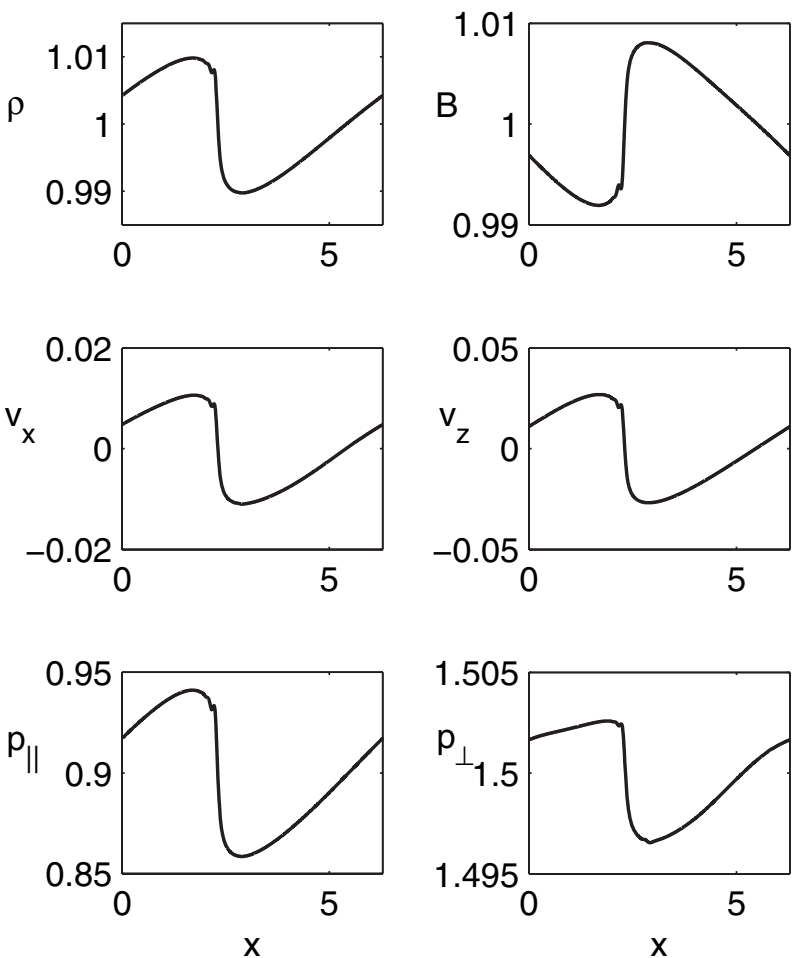

Fig. 5. Spatial profiles of various quantities at $t=28 t_{A}$ for the nonlinear slow waves with $(\omega / k)_{s}>(\omega / k)_{i}$ and $\delta B \delta \rho<0$; the parameter values used are: $\beta_{\perp, i}=3, \beta_{\|, i}=1.8, \theta=40^{0}$, and $\gamma_{\perp, i}=2$, $\gamma_{\|, i}=3$.

these anomalous slow waves can actually exist in the nonlinear model. Recently the formation of anomalous slow shocks is examined by means of the MHD Riemann problem (Hau and Hung, 2005). To investigate this problem further, the linear anomalous slow waves (in the fire-hose and mirror stable parameter regimes) are used as the initial conditions of the nonlinear model to allow for full evolution. Figure 5 shows the spatial profiles of all physical quantities at $t=28 t_{A}$ for the case where $(\omega / k)_{s}>(\omega / k)_{i}$ and $\delta B \delta \rho<0$. Figure 6 shows similar results for the case where $(\omega / k)_{s}>(\omega / k)_{i}$ and $\delta B \delta \rho>0$. In both cases the anisotropic MHD jump relations (Hau and Hung, 2005) are examined in the shock moving frame and found to be well satisfied. In addition, the flow velocities in the upstream and downstream of the shocks are found to be super-slow, super-Alfvénic, sub-fast and sub-slow, super-Alfvénic that the shocks can be classified as super-Alfvénic slow shocks. Numerical calculations using various parameter values and polytropic exponents also show similar results, i.e., stable anomalous slow waves with $(\omega / k)_{s}>(\omega / k)_{i}$ and $\delta B \delta \rho<0$ or $\delta B \delta \rho>0$ may tend to steepen into anomalous slow shocks in the nonlinear model.


Fig. 6. Spatial profiles of various quantities at $t=25 t_{A}$ for the nonlinear slow waves with $(\omega / k)_{s}>(\omega / k)_{i}$ and $\delta B \delta \rho>0$; the parameter values used are: $\beta_{\perp, i}=1.6, \beta_{\|, i}=3.4, \theta=40^{0}$, and $\gamma_{\perp, i}=2$, $\gamma_{\|, i}=3$.

\subsection{Fire-hose instability}

In our earlier study of nonlinear fire-hose instabilities, the focus has been on the comparison between MHD and kinetic results of standard fire-hose instability and the competition between incompressible and compressible fire-hose instabilities. In the present study we examine the nonlinear evolution of standard and compressible fire-hose instabilities individually for various parameter values. The initial perturbations are the linear incompressible or compressible firehose modes. Figure 7 shows the spatial profiles of various physical quantities at various times for the parameter values of $\beta_{\|, i}=8.5, \beta_{\perp, i}=6, \gamma_{\|, i}=1 / 2, \gamma_{\perp, i}=2$, and $\theta=10^{0}$. This case corresponds to the normal compressible fire hose in that the intermediate fire hose grows faster than the compressible fire hose. It is seen that the compressible fire hose with $\gamma_{i}>\gamma_{s}$ alone behaves differently from the case mixed with the intermediate fire hose (Fig. 4 of Wang and Hau, 2003). In particular, an initially sinusoidal slow wave will evolve to the state with two magnetic field dips associated with increased density where the plasma $\beta$ values and the quantity, $\beta_{\|, i}-\beta_{\perp, i}$, are relatively large. Outside the magnetic dips, the marginal fire-hose stability threshold, $\beta_{\|, i}-\beta_{\perp, i}=2$, is more or less reached. Examining the propagation angle and 
$\rho$
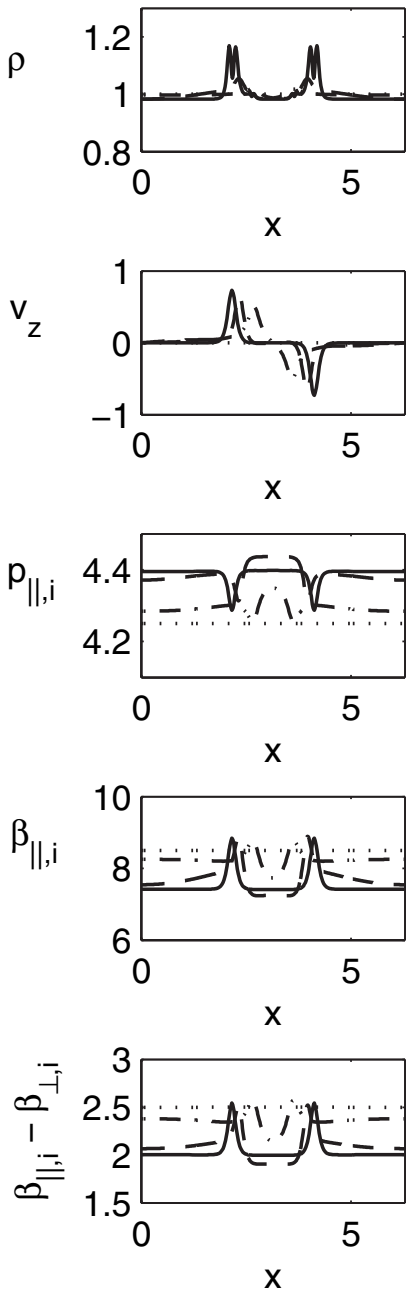

$\mathrm{B}$
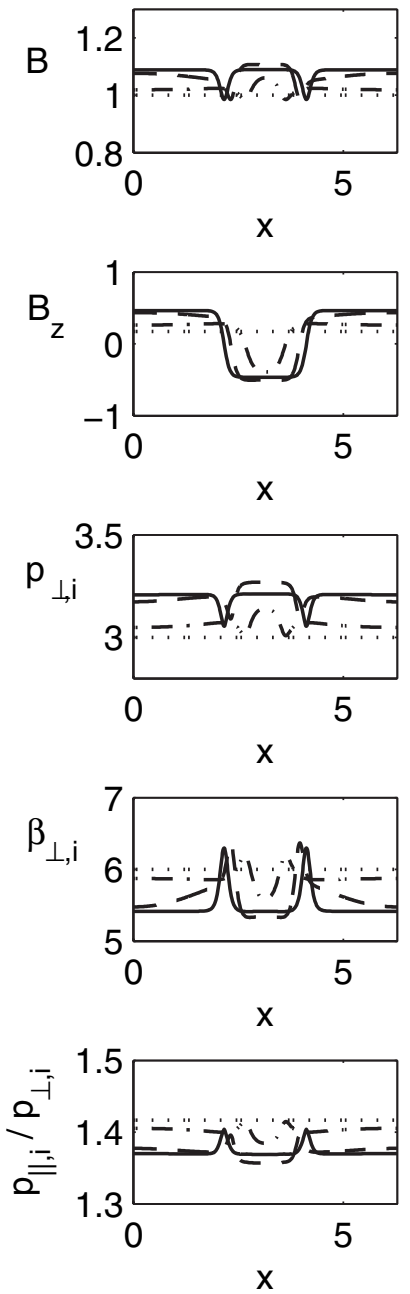

Fig. 7. Time evolution of various quantities at $t=0$ (dotted line), $25 t_{A}$ (dash-dotted line), $30 t_{A}$ (dashed line), and $60 t_{A}$ (solid line) for compressible fire-hose instability with $\gamma_{i}>\gamma_{s}$; the parameter values used are: $\beta_{\|, i}=8.5, \beta_{\perp, i}=6, \gamma_{\|, i}=1 / 2, \gamma_{\perp, i}=2$, and $\theta=10^{0}$.

the critical angle for fire-hose instability to occur (Eq. 13) shows that $\theta>\theta_{c}$ is reached at the nonlinear saturated state despite that the necessary (but not sufficient) instability condition, $\beta_{\|, i}-\beta_{\perp, i}>2$, still prevails in the magnetic dip region.

Figure 8 shows similar calculations for the parameter values of $\beta_{\|, i}=8.1, \beta_{\perp, i}=6, \gamma_{\|, i}=1 / 2, \gamma_{\perp, i}=1\left(\gamma_{\|, i} \gamma_{\perp, i}<1\right)$, and $\theta=35^{0}$ that corresponds to the anomalous case of $\gamma_{s}>\gamma_{i}$ and thus complies with the mirror criterion of Eq. (12). In contrast with the previous case where the magnetic field is increased in the entire domain, a region of depressed magnetic field accompanied by an enhanced density is developed and the nonlinear saturated state is well below the standard fire-hose condition of $\beta_{\|, i}-\beta_{\perp, i}=2$. The result that a region of pronounced weak magnetic field may form and the $\left(\beta_{\|, i}-\beta_{\perp, i}\right)$ value of the saturated state can possibly be lower than 2 is due to the fact that the mirror quantity $m=\left(p_{\perp} / p_{\|}-\gamma_{\|}\left(\gamma_{\perp}+2 / \beta_{\perp}\right)\right)$ is increased with time
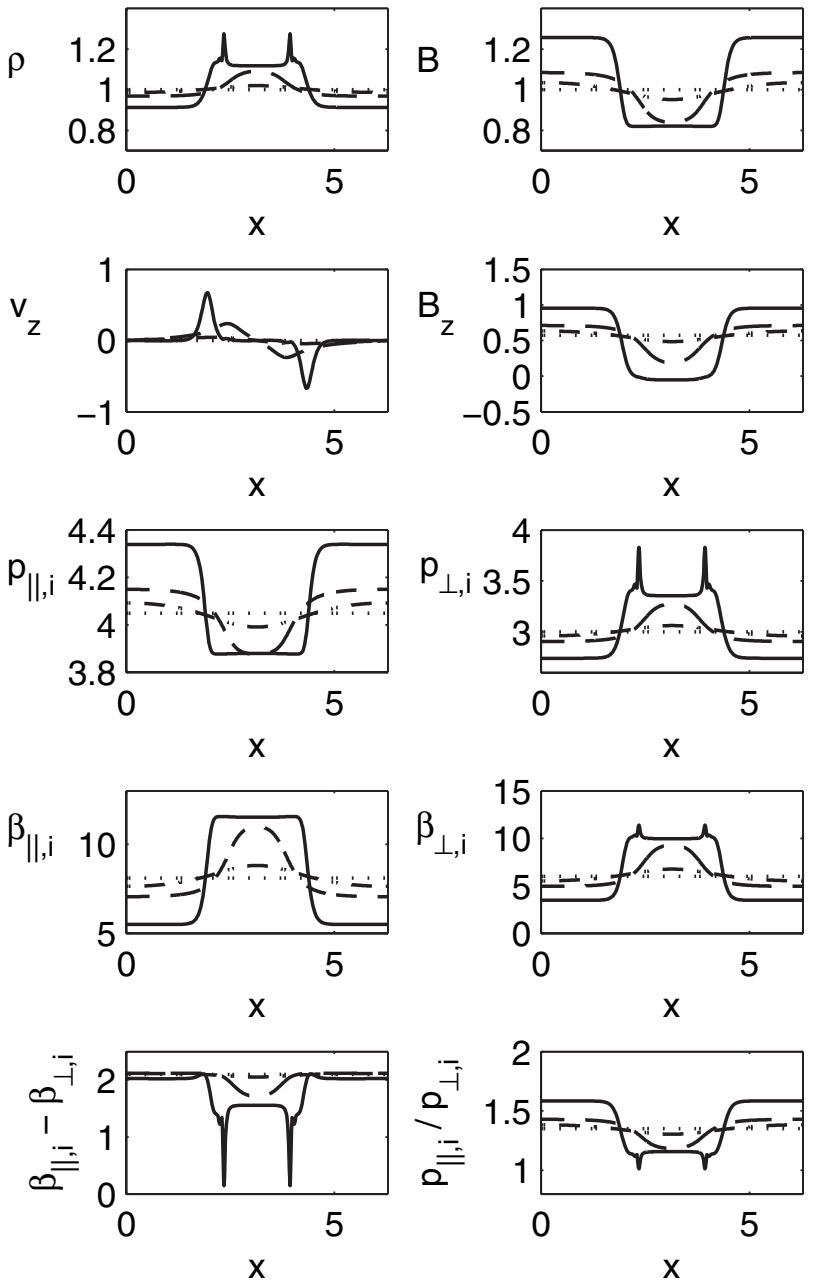

Fig. 8. Time evolution of various quantities at $t=0$ (dotted line), $30 t_{A}$ (dash-dotted line), $35 t_{A}$ (dashed line), and $50 t_{A}$ (solid line) for compressible fire-hose instability with $\gamma_{s}>\gamma_{i}$; the parameter values used are: $\beta_{\|, i}=8.1, \beta_{\perp, i}=6, \gamma_{\|, i}=1 / 2, \gamma_{\perp, i}=1$, and $\theta=35^{0}$.

within the magnetic depression where both quantities, $\beta_{\perp}$ and $p_{\perp} / p_{\|}=B^{\gamma_{\|}+\gamma_{\perp}-2} \rho^{1-\gamma_{\|}}$(obtained from the combined double-polytropic laws), are increased with time. The nonlinear saturation of this anomalous slow fire hose is found to comply with the linear mirror stability criteria (Wang and Hau, 2003); in particular, although the condition of $p_{\perp} / p_{\|}>\gamma_{\|}\left(\gamma_{\perp}+2 / \beta_{\perp}\right)$ is not satisfied but the propagation angle becomes more parallel due to decreased $B_{z}$ such that $\theta<\theta_{c}$. This latter condition is not established initially because of $\beta_{\|, i}-\beta_{\perp, i}>2$ until the instability evolves toward the state of $\beta_{\|, i}-\beta_{\perp, i}<2$ where $\theta_{c}$ in Eq. (13) becomes physical and both the mirror conditions of Eqs. (12) and (13) shall be complied for the compressible fire hose with $\gamma_{\|, i} \gamma_{\perp, i}<1$ to be unstable.

In the cases shown in Figs. 7 and 8, the standard firehose criterion, $\beta_{\|, i}-\beta_{\perp, i}>2$, is satisfied that the intermediate mode is indeed also unstable. For comparison, Figs. 9 and 10 

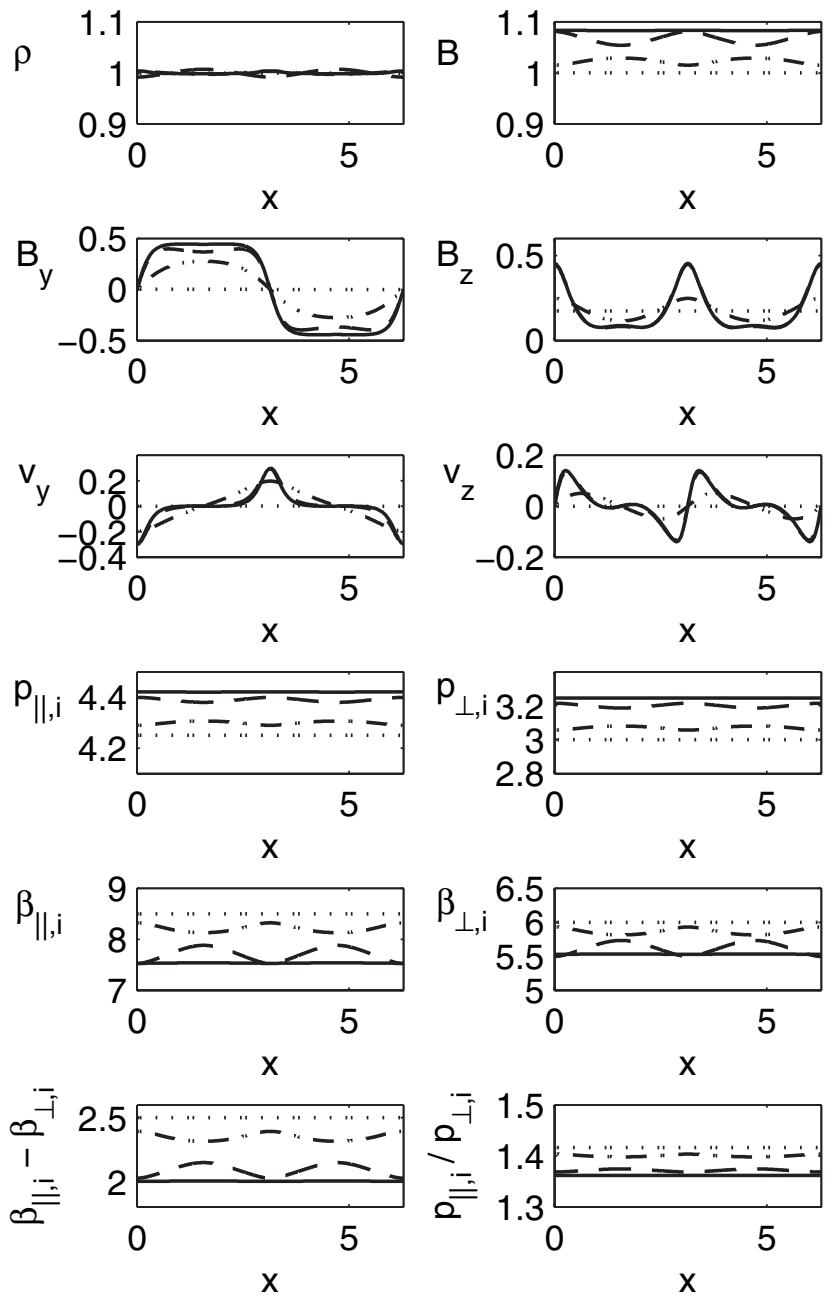

Fig. 9. Time evolution of various quantities at $t=0$ (dotted line), $20 t_{A}$ (dash-dotted line), $30 t_{A}$ (dashed line), and $60 t_{A}$ (solid line) for standard fire-hose instability with $\gamma_{i}>\gamma_{s}$; the parameter values used are: $\beta_{\|, i}=8.5, \beta_{\perp, i}=6, \gamma_{\|, i}=1 / 2, \gamma_{\perp, i}=2$, and $\theta=10^{0}$.

show two cases of the intermediate fire hose with the same parameter values as in Figs. 7 and 8 (except for $\theta=10^{0}$ in Fig. 10). The example shown in Fig. 9 has $\gamma_{i}>\gamma_{s}$ such that the dynamics is controlled primarily by the incompressible fire-hose mode and thus there exist no pronounced magnetic field and density fluctuations in the nonlinear phase. In this case the standard fire-hose criterion, $\beta_{\|, i}-\beta_{\perp, i}=2$, is satisfied in the entire domain. The example shown in Fig. 10 has the property of $\gamma_{s}>\gamma_{i}$ that the compressible fire hose may enter the dynamics though the initial perturbations are of pure incompressible fire-hose mode. As a result, in contrast with Fig. 9, there are clear signatures of magnetic field and density perturbations and the quantity, $\beta_{\|, i}-\beta_{\perp, i}$, may be less than 2 as the case shown in Fig. 8. In all the cases shown in Figs. 8-10, the nonlinear states remain to be of fire-hose type pressure anisotropy of $p_{\|}>p_{\perp}$ complying with standard fire-hose or generalized mirror stability criteria.
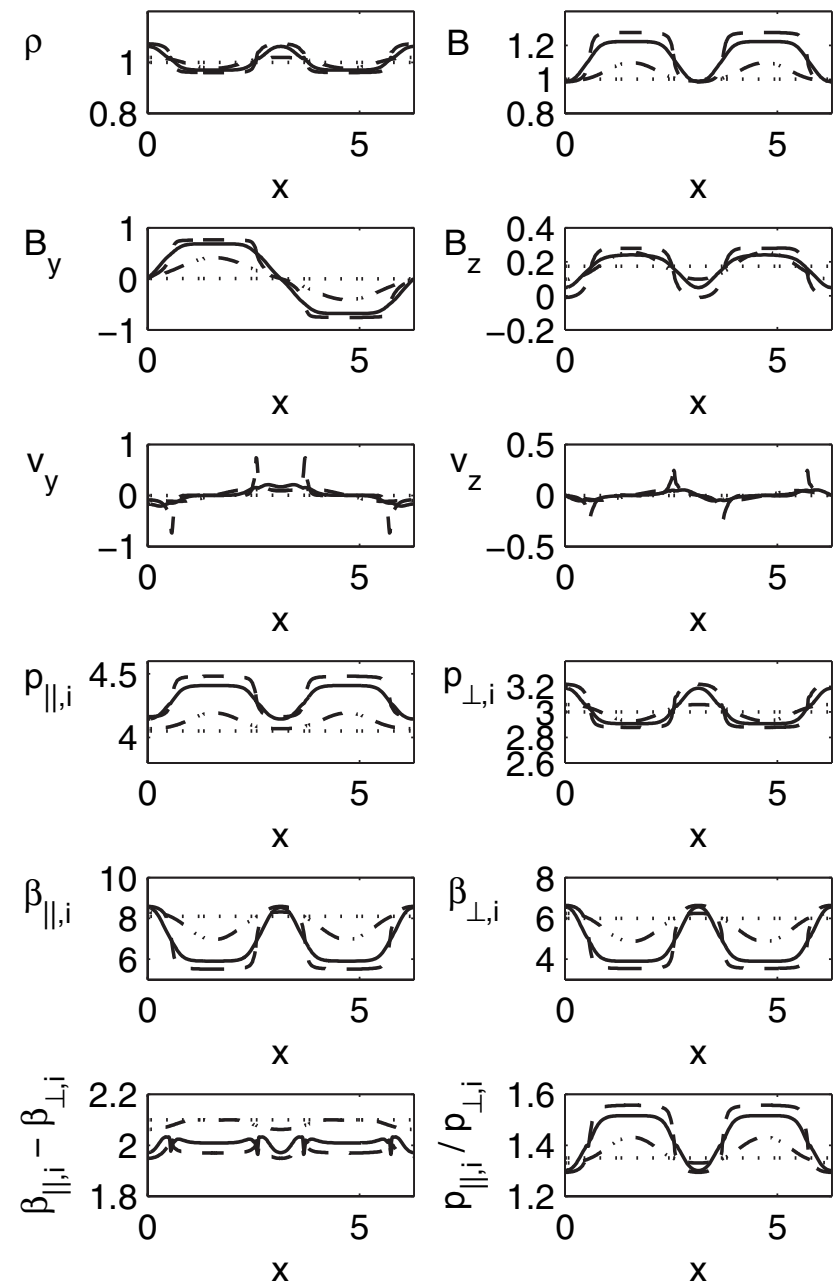

Fig. 10. Time evolution of various quantities at $t=0$ (dotted line), $60 t_{A}$ (dash-dotted line), $70 t_{A}$ (dashed line), and $80 t_{A}$ (solid line) for standard fire-hose instability with $\gamma_{s}>\gamma_{i}$; the parameter values used are: $\beta_{\|, i}=8.1, \beta_{\perp, i}=6, \gamma_{\|, i}=1 / 2, \gamma_{\perp, i}=1$, and $\theta=10^{0}$.

\subsection{Mirror instability}

As compared to the fire-hose instability, mirror instability has been studied extensively since its early discovery because of frequent observations of nonlinear structures with depressed magnetic field accompanied by enhanced plasma density in the planetary magnetospheres (e.g., Hasegawa, 1969; Tsurutani et al., 1982; Pokhotelov et al., 2004). The study of nonlinear mirror instability however is mostly based on the kinetic theory and particle simulation (e.g., McKean et al., 1994). In the present study simple gyrotropic MHD model is adopted to examine the nonlinear evolution of mirror instability. As in the previous two sections, various perturbations derived from the linear double-polytropic MHD model are used as the initial conditions of the nonlinear numerical model to allow for the full evolution of mirror instability. Figure 11 shows the spatial profiles of various quantities 

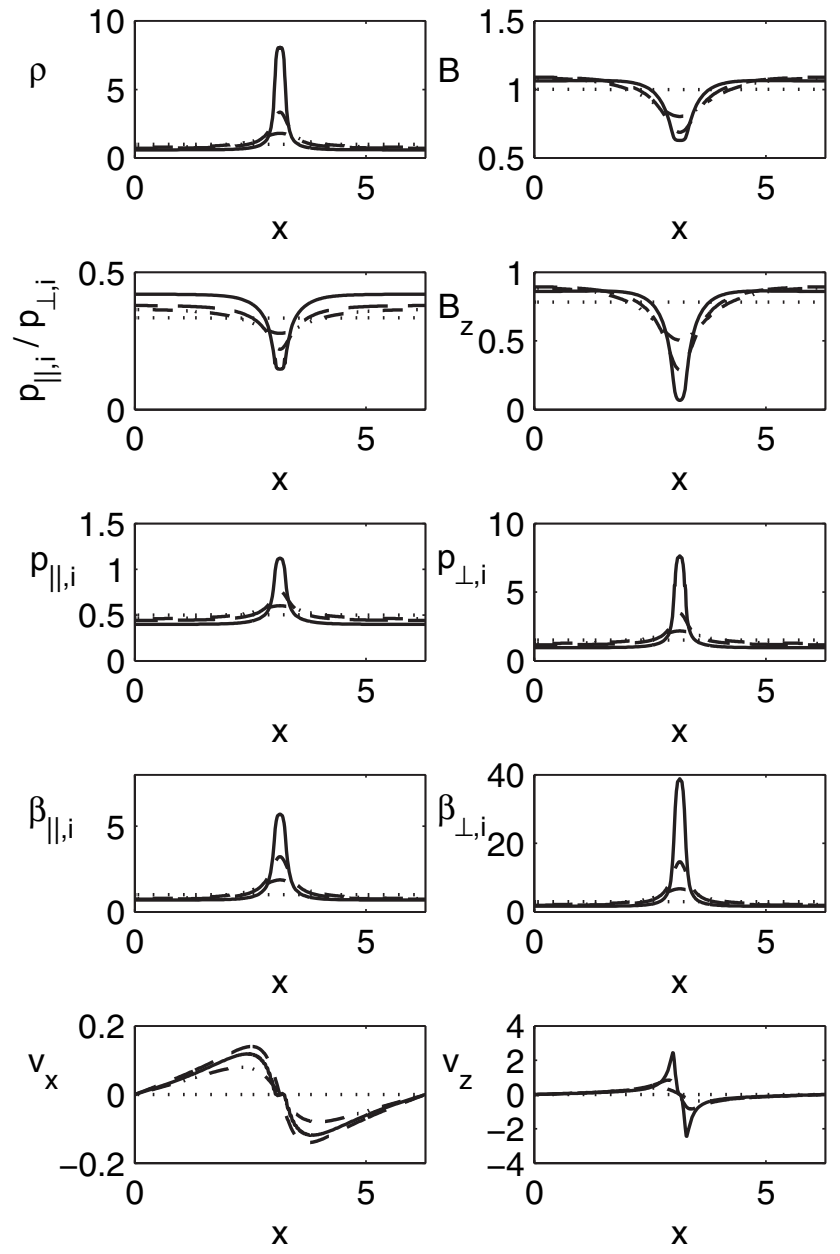

Fig. 11. Time evolution of various quantities at $t=0$ (dotted line), $40 t_{A}$ (dash-dotted line), $42 t_{A}$ (dashed line), and $45 t_{A}$ (solid line) for the mirror instability; the parameter values used are: $\beta_{\|, i}=1, \beta_{\perp, i}=3, \gamma_{\|, i}=1 / 2, \gamma_{\perp, i}=2$, and $\theta=51.4^{0}$.

at various times for the parameter values of $\beta_{\|, i}=1, \beta_{\perp, i}=3$, $\gamma_{\|, i}=1 / 2, \gamma_{\perp, i}=2$, and $\theta=51.4^{0}$. It is seen that a region of depressed magnetic field accompanied by a pronounced density enhancement forms from an initially sinusoidal unstable slow/mirror wave. The formation process of the magnetic hole in the mirror instability is indeed similar to that of the slow fire-hose instability shown in Fig. 8. Specifically, the decreased magnetic field along with increased plasma density will lead to further increase of $\beta_{\perp}$ and $p_{\perp} / p_{\|}$, and the mirror condition, $m=\left(p_{\perp} / p_{\|}-\gamma_{\|}\left(\gamma_{\perp}+2 / \beta_{\perp}\right)\right)>0$, is thus further violated. Figure 12 shows the time evolution of the mirror quantity $m$ and the propagation angle $\theta$, between wave vector and magnetic field, as well as the critical angle $\theta_{c}$ in Eq. (13) for the entire simulation region. As indicated, within the magnetic depression region the propagation angle $\theta$ is gradually decreased with time such that eventually $\theta<\theta_{c}$ and the instability ceases to grow despite the mirror stability condition, $m \leq 0$, is far from being satisfied.
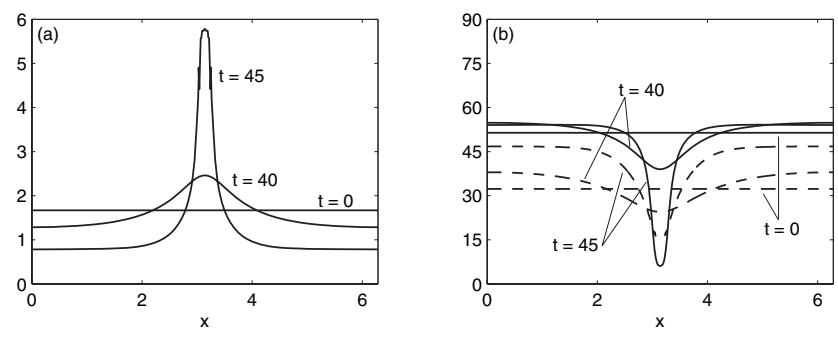

Fig. 12. Time development of (a) the mirror instability quantity $m=\left(p_{\perp} / p_{\|}-\gamma_{\|}\left(\gamma_{\perp}+2 / \beta_{\perp}\right)\right)(m<0$ is for stability) and (b) the propagation angle (solid curves) as well as the critical angle in Eq. (13) (dashed curves) for the mirror instability shown in Fig. 11. The various curves in each panel are for $t=0,40 t_{A}$, and $45 t_{A}$.

\section{Discussion and summary}

In this paper the properties of MHD waves, fire-hose and mirror instabilities in gyrotropic plasmas are examined systematically within the framework of generalized CGL-MHD model. Due to profound difficulty with the energy closure in anisotropic plasmas (e.g., Ferrière and André, 2002), a set of empirical laws with two polytropic exponents are used to describe the dynamical evolution of parallel and perpendicular pressures. The model enables the distinct properties of ion and electron fluids, such as the pressure anisotropy, plasma beta and polytropic exponents etc, to be taken into account. The present study on the properties of linear MHD waves and instabilities is an extension of our earlier work based on the one-fluid MHD model (Hau and Sonnerup, 1993; Wang and Hau, 2003). The conditions for the occurrence of anomalous slow waves with phase speed greater than that of intermediate waves and/or with positive density-magnetic field correlation are obtained. Moreover, the general conditions for the occurrence of fire-hose and mirror instabilities are examined thoroughly within the framework of double-polytropic MHD model. It is shown that the intermediate mode may develop standard fire-hose instability while the slow mode may develop not only mirror instability but also a new type of compressible fire-hose instability. This property is the profound characteristic of the gyrotropic MHD model and may occur for various polytropic exponents though for cold electrons and $\gamma_{\|, i}=1 / 2, \gamma_{\perp, i}=2$ the stability criteria are identical to those derived from the Vlasov theory. For the slow fire hose to grow faster than the intermediate fire hose there is constraint on the ion polytropic values for given other parameter values. The significance of the results derived from the MHD theory is that the new type of compressible firehose instability and the possibility that it may grow faster than the standard fire hose are originally found in the kinetic model (Hellinger and Matsumoto, 2000, 2001). The MHD model however offers a much simple picture for the existence and characteristics of compressible fire-hose instability and its relation to various MHD wave modes. Two 
kinds of compressible fire-hose instabilities are identified in the MHD model complying with different instability criteria, the standard fire-hose and generalized mirror conditions. For the latter case, the compressible fire-hose and mirror instabilities not only originate from the same mode but also obey the same stability criteria though the nature of the pressure anisotropy is of $p_{\|}>p_{\perp}$ and $p_{\perp}>p_{\|}$, respectively, and the polytropic values are not the same.

Taking advantage of the result that the linear stability criteria for both fire-hose and mirror instabilities may recover those derived from the kinetic theory, the characteristics of slow waves, fire-hose and mirror instabilities are further examined by the numerical double-polytropic MHD simulations. The experiments of nonlinear evolution of stable anomalous slow waves show that anomalous slow shocks may form through the steepening process of anomalous slow waves. These anomalous slow shocks may travel faster than the intermediate wave with upstream and downstream flows being super-slow, super-Alfvénic and sub-slow but superAlfvénic, respectively, and/or may possess positive densitymagnetic field correlation like the fast shock. The results are consistent with those obtained from the different experiments conducted by Hau and Hung (2005) for which the anomalous slow shocks are generated by imposing a magnetic normal on an initially tangential discontinuity current layer. Observational evidences for slow shocks moving ahead of the rotational discontinuity at the magnetopause and in the solar wind have been found by Walthour et al. (1994) and Whang et al. (1998). Our calculations provide a possible explanation for the observed abnormal behaviors of MHD discontinuities. The result that slow shocks may possess positive density-magnetic field correlation also has important implications to space plasma observations; in particular, in the presence of temperature anisotropy the density and magnetic field correlation may not be used as a single criterion for distinguishing slow shocks from fast shocks in the observational data.

The numerical experiments of nonlinear evolution of firehose instabilities show that the regular slow fire hose complying with the standard fire-hose instability criterion of $\beta_{\|, i}-\beta_{\perp, i}>2$ and the abnormal slow fire hose complying with the generalized mirror criteria may develop regions of weak magnetic field associated with enhanced plasma density. This result is in contrast with the standard fire-hose instability which does not exhibit any pronounced magnetic field and density fluctuations. All three kinds of fire-hose instabilities are found to saturate at different stability conditions; in particular, the standard fire hose behaves quasilinearly in that the nonlinear state satisfies more or less the linear stability criterion of $\beta_{\|, i}-\beta_{\perp, i}=2$, a result consistent with the particle simulations. The normal compressible firehose instability however saturates at slightly different conditions in that the threshold of $\beta_{\|, i}-\beta_{\perp, i}=2$ is not reached but the propagation angle becomes more oblique to satisfy the necessary condition of $\theta<\theta_{c}$ for compressible fire-hose instability. The nonlinear saturation of abnormal compressible fire-hose instability also does not satisfy its linear stability condition of $p_{\perp} / p_{\|}=\gamma_{\|}\left(\gamma_{\perp}+2 / \beta_{\perp}\right)$ but do meet the requirement of $\theta<\theta_{c}$ for the instability to cease growing. This abnormal fire-hose instability not only has larger linear growth rate than the standard fire hose but also saturates at $\beta_{\|, i}-\beta_{\perp, i}<2$ and is thus more effective at destroying the firehose type pressure anisotropy than the standard fire hose, a result first found in the hybrid particle simulations (Hellinger and Matsumoto, 2000).

One major triumph with the double-polytropic MHD model is that the derived generalized mirror instability criteria in the limit of cold electrons and $\gamma_{\|, i}=1 / 2, \gamma_{\perp, i}=2$ are identical to the kinetic result. It is thus worthwhile to examine the nonlinear evolution of mirror instability based on the simple gyrotropic MHD model. The calculations show that the trough region of the magnetic field associated with enhanced plasma density may fully develop in the nonlinear regime and the formation mechanism for magnetic holes is similar to the abnormal fire-hose instability in that in the weak field region the mirror stability criterion is violated further as the instability develops due to increased $\beta_{\perp}$ and $p_{\perp} / p_{\|}$. The nonlinear saturated state is thus far from satisfying the mirror threshold of $\beta_{\|}=\beta_{\perp}^{2} /\left(1+\beta_{\perp}\right)$, but rather it falls in the regime of $\theta<\theta_{c}$ due to decreased transverse magnetic field. Specifically, the mirror instability becomes saturated when the propagation angle becomes less oblique to satisfy the necessary condition, $\theta>\theta_{c}$, for linear mirror instability. In this regard, the nonlinear saturation of both compressible fire-hose and mirror instabilities indeed complies with the linear stability thresholds and it is not incorrect to conclude that they behave quasi-linearly. Nevertheless our result that the nonlinear compressible fire-hose and mirror instabilities do not comply with the standard fire-hose and mirror thresholds of $\beta_{\|, i}-\beta_{\perp, i}=2$ and $\beta_{\|}=\beta_{\perp}^{2} /\left(1+\beta_{\perp}\right)$, respectively, agrees with the kinetic simulations.

The mirror instability has been considered as the likely process for magnetic hole formation and structures observed in the space plasma environment (see an overview by Tsurutani et al., 2005) though other mechanisms, such as Alfvén solitons, slow-mode shocks, nonlinear Alfvén waves and slow solitons proposed by various authors (Baumgärtel, 1999; Farrugia et al., 2001; Buti et al., 2001; Tsurutani et al., 2002; Dasgupta et al., 2003; Stasiewicz, 2004) may also lead to similar hole structures with depressed magnetic field. Identification of different mechanisms in the observational data however is nontrivial because of their similarity; in particular, the density and magnetic field features associated with the mirror instability shown in Fig. 11 may appear like those of slow solitons proposed by Stasiewicz (2004) as a possible theoretical model for magnetic hole structures observed in the magnetopause and boundary layers by the Cluster spacecraft (Stasiewicz et al., 2003). The formation mechanism for slow solitons and nonlinear mirror structures however is different; specifically, the former is a nondissipative 
structure and the dispersive effect such as the Hall current rather than the pressure anisotropy is essential while the latter ones involve temperature anisotropy and may form in the dissipative, nondispersive MHD model as shown here. As pointed out by Hau et al. (2005), in the context of gyrotropic Hall MHD model these two structures may appear similar. Studies of nonlinear mirror instability in the gyrotropic Hall MHD model will be carried out in the future to further examine the problem.

Acknowledgements. This research is supported by the National Science Council of the Republic of China under grants NSC952111-M-008-001 and NSC96-2111-M-008-001 to National Central University.

Edited by: B. Tsurutani

Reviewed by: two anonymous referees

\section{References}

Anderson, B. J., Engerbretson, M. J., Rounds, S. P., Zanetti, L. J., and Potmera, T. A.: A statistical study of Pc 3-5 pulsations observed by AMPTE/CCE magnetic field experiment: 1. Occurrence distributions, J. Geophys. Res., 95, 10495, 1990.

Baumgärtel, K.: Soliton approach to magnetic holes, J. Geophys. Res., 104, 28 295-28 308, 1999.

Buti, B., Tsurutani, B. T., Neugebauer, M., and Goldstein, B. E.: Generation mechanism for magnetic holes in the solar wind, Geophys. Res. Lett., 28, 1355-1358, 2001.

Chew, G. F., Goldberger, M. L., and Low, F. E.: The Boltzmann equation and the one-fluid hydromagnetic equations in the absence of particle collisions, Proc. Roy. Soc. London, Ser A, 236, $112-118,1956$.

Crooker, N. U. and Siscoe, G. L.: A mechanism for pressure anisotropy and mirror instability in the dayside magnetopause, J. Geophys. Res., 82, 185, 1977.

Dasgupta, B., Tsurutani, B. T., and Janaki, M. S.: A kinetic approach to the Pondermotive Force, Geophys. Res. Lett., 30, 2128 , doi:10.1029/2003GL017385, 2003.

Farrugia, C. J., Vasquez, B., Richardson, I. G., Torbert, R. B., Burlaga, L. F., Biernat, H. K., Mühlbachler, S., Ogilvie, K. W., Lepping, R. P., Scudder, J. D., Berdichevsky, D. E., Semenov, V. S., Kubyshkin, I. V., Phan, T. D., and Lin, R. P.: A reconnection layer associated with a magnetic cloud, Adv. Space Res., 28, 759-764, 2001.

Ferrière, K. M. and André, N.: A mixed magnetohydrodynamickinetic theory of low-frequency waves and instabilities in homogeneous, gyrotropic plasmas, J. Geophys. Res., 107, 1349, doi:10.1029/2002JA009273, 2002.

Greenstadt, E. W., Green, I. M., and Inouye, G. T.: Correlated magnetic field and plasma observations of the Earth's bow shock, J. Geophys. Res., 73, 51-60, 1968.

Gary, S. P., Li, H., O'Rourke, S., and Winske, D.: Proton resonant firehose instability: Temperature anisotropy and fluctuating field constraints, J. Geophys. Res., 103, 14 567-14 574, 1998.

Hasegawa, A.: Drift mirror instability in the magnetosphere, Phys. Fluids, 12, 2642-2650, 1969.
Hasegawa, A.: Plasma Instabilities and Nonlinear Effects, p. 94, Springer-Verlag, New York, 1975.

Hada, T. and Kennel, C. F.: Nonlinear evolution of slow waves in the solar wind, J. Geophys. Res., 90, 531-535, 1985.

Hau, L.-N. and Sonnerup, B. U. Ö.: On slow mode waves in anisotropic plasmas, Geophys. Res. Lett., 20, 1763-1766, 1993.

Hau, L.-N., Phan, T.-D., Sonnerup, B. U. Ö., and Paschmann, G.: Double-polytropic closure in the magnetosheath, Geophys. Res. Lett., 20, 2255-2258, 1993.

Hau, L.-N.: Nonideal MHD effects in the magnetosheath, J. Geophys. Res., 101, 2655-2660, 1996.

Hau, L.-N.: A note on the energy laws in gyrotropic plasmas, Phys. Plasmas, 9, 2455-2457, 2002.

Hau, L.-N. and Hung, C.-C.: Formation of anomalous slow shocks in anisotropic plasmas, Geophys. Res. Lett., 32, L14103, doi:10.1029/2005GL023167, 2005.

Hau, L.-N., Wang, B.-J., and Teh, W. L.: Slow mode waves and mirror instability in gyrotropic Hall magnetohydrodynamic model, Phys. Plasmas, 12, 122904, 2005.

Hellinger, P. and Matsumoto, H.: New kinetic instability: Oblique Alfvén fire hose, J. Geophys. Res., 105, 10 519-10 526, 2000.

Hellinger, P. and Matsumoto, H.: Nonlinear competition between the whistler and Alfvén fire hoses, J. Geophys. Res., 106, 13 215-13 218, 2001.

Hellinger, P., Trávníek, P., Kasper, J. C., and Lazarus, A. J.: Solar wind proton temperature anisotropy: linear theory and WIND/SWE observations, Geophys. Res., Lett., 33, L09101, doi:10.1029/2006GL025925, 2006.

Hill, P., Paschmann, G., Treumann, R. A., Baumjohann, W., Sckopke, N., and Luhr, H.: Plasma and magnetic field behavior across the magnetosheath near local noon, J. Geophys. Res., 100, 9575-9584, 1995.

Kivelson, M. G. and Southwood, D. J.: Mirror instability II: The mechanism of nonlinear saturation, J. Geophys. Res., 101, 17365-17 371, 1996.

Kasper, J. C. and Lazarus, A. J.: Wind/SWE observations of firehose constraint on solar wind proton temperature anisotropy, Geophys. Res. Lett., 29, 1839, doi:10.1029/2002GL015128, 2002.

McKean, M. E., Winske, D., and Gary, S. P.: Two-dimensional simulations of ion anisotropy instabilities in the magnetosheath, J. Geophys. Res., 99, 11 141-11 153, 1994.

Phan, T. D., Larson, D. E., Lin, R. P., McFadden, J. P., Anderson, K. A., Carlson, C. W., Ergun, R. E., Ashford, S. M., McCarthy, M. P., Parks, G. K., Reme, H., Bosqued, J. M., D’Uston, C. D., Wenzel, K.-P., Sanderson, T. R., and Szabo, A.: The subsolar magnetosheath and magnetopause for high solar wind ram pressure: WIND observations, Geophys. Res. Lett., 23, 1279-1282, 1996.

Pokhotelov, O. A., Sagdeev, R. Z., Balikhin, M. A., and Treumann, R. A.: Mirror instability at finite ion-Larmor radius wavelengths, J. Geophys. Res., 109, A09213, doi:10.1029/2004JA010568, 2004.

Quest, K. B. and Shapiro, V. D.: Evolution of the fire-hose instability: Linear theory and wave-wave coupling, J. Geophys. Res., 101, 24457-24469, 1996.

Stasiewicz, K., Shukla, P. K., Gustafsson, G., Buchert, S., Lavraud, B., Thidé, B., and Klos, Z.: Slow magnetosonic solitons detected by the Cluster spacecraft, Phys. Rev. Lett., 90, 085002, 2003. 
Stasiewicz, K.: Theory and observations of slow-mode solitons in space plasmas, Phys. Rev. Lett., 93, 125004, 2004.

Tsurutani, B. T. and Rodriguez, P.: Upstream waves and particles: An overview of ISEE results, J. Geophys. Res., 86, 4319-4324, 1981.

Tsurutani, B. T, Smith, E. J., Anderson, R. R., Ogilvie, K. W., Scudder, J. D., Baker, D. N., and Bame, S. J.: Lion roars and nonoscillatory drift mirror waves in the magnetosheath, J. Geophys. Res., 87, 6060-6072, 1982.

Tsurutani, B. T., Southwood, D. J., Smith, E. J., and Balogh, A.: Nonlinear magnetosonic waves and mirror mode structures in the March 1991 Ulysses interplanetary event, Geophys. Res. Lett., 19, 1267-1270, 1992.

Tsurutani, B. T., Galvan, C., Arballo, J. K., Winterhalter, D., Sakurai, R., Smith, E. J., Buti, B., Lakhina, G. S., and Balogh, A.: Relationship between discontinuities, magnetic holes, magnetic decreases, and nonlinear Alfvén waves: Ulysses observations over the solar poles, Geophys. Res. Lett., 29, 23-1, 1528, doi:10.1029/2001GL013623, 2002.
Tsurutani, B. T., Lakhina, G. S., Pickett, J. S., Guarnieri, F. L., Lin, N., and Goldstein, B. E.: Nonlinear Alfvén waves, discontinuities, proton perpendicular acceleration, and magnetic holes/decreases in interplanetary space and the magnetosphere: intermediate shocks?, Nonlin. Processes Geophys., 12, 321-336, 2005 ,

http://www.nonlin-processes-geophys.net/12/321/2005/.

Walthour, D. W., Gosling, J. T., Sonnerup, B. U. Ö., and Russell, C. T.: Observation of anomalous slow-mode shock and reconnection layer in the dayside magnetopause, J. Geophys. Res., 99, 23 705-23 722, 1994.

Whang, Y. C., Zhou, J., Lepping, R. P., Szabo, A., Fairfield, D., Kokubum, S., Ogilvie, K. W., and Fitzenreiter, R.: Double discontinuity: A compound structure of slow shock and rotational discontinuity, Geophys. Res. Lett., 24, 3153-3156, 1998.

Wang, B.-J. and Hau, L.-N.: MHD aspects of fire-hose type instabilities, J. Geophys. Res., 108, 1463, doi:10.1029/2003JA009986, 2003. 